\title{
Comfort-driven disparity adjustment for stereoscopic video
}

\author{
Miao Wang ${ }^{1}(\bigotimes)$, Xi-Jin Zhang ${ }^{1}$, Jun-Bang Liang ${ }^{1}$, Song-Hai Zhang ${ }^{1}$, and Ralph R. Martin ${ }^{2}$ \\ (c) The Author(s) 2016. This article is published with open access at Springerlink.com
}

Abstract Pixel disparity - the offset of corresponding pixels between left and right views - is a crucial parameter in stereoscopic three-dimensional (S3D) video, as it determines the depth perceived by the human visual system (HVS). Unsuitable pixel disparity distribution throughout an S3D video may lead to visual discomfort. We present a unified and extensible stereoscopic video disparity adjustment framework which improves the viewing experience for an S3D video by keeping the perceived $3 \mathrm{D}$ appearance as unchanged as possible while minimizing discomfort. We first analyse disparity and motion attributes of S3D video in general, then derive a wide-ranging visual discomfort metric from existing perceptual comfort models. An objective function based on this metric is used as the basis of a hierarchical optimisation method to find a disparity mapping function for each input video frame. Warping-based disparity manipulation is then applied to the input video to generate the output video, using the desired disparity mappings as constraints. Our comfort metric takes into account disparity range, motion, and stereoscopic window violation; the framework could easily be extended to use further visual comfort models. We demonstrate the power of our approach using both animated cartoons and real S3D videos.

Keywords stereoscopic video editing; video enhancement; perceptual visual computing; video manipulation

1 Department of Computer Science and Technology, Tsinghua University, Beijing 100084, China. E-mail: M. Wang, wangmiao11@mails.tsinghua.edu.cn (西); X.-J. Zhang, zhangxijin91@gmail.com; J.-B. Liang, williamm 2006@126.com; S.-H. Zhang, shz@tsinghua.edu.cn.

2 Cardiff University, Cardiff, CF243AA, UK. E-mail: ralph@cs.cf.ac.uk.

Manuscript received: 2015-12-02; accepted: 2015-12-09

\section{Introduction}

With the recent worldwide increase in stereoscopic display hardware, there has been great interest in both academia and industry in stereoscopic threedimensional (S3D) movie production, for instance, glasses-free multi-view display technology $[1,2]$ and perceptual disparity models [3, 4]. Viewing the 3D world through a display screen differs from natural viewing - it introduces vergenceaccommodation conflict $[5,6]$. As a result, poor scene design in S3D movies can lead to visual fatigue. In addition to vergence-accommodation conflict, other factors such as motion and luminance also affect the human visual system (HVS), and may make the viewer feel uncomfortable. Most of these factors have a close relationship to binocular disparity - the difference in an object's location on the left and right retinas [7]. The brain uses binocular disparity to extract depth information via a process of stereopsis.

The goal of making a movie stereoscopic is to add realism by providing a feeling of depth, but care must be taken to avoid visual discomfort. It is thus a tedious process to tune the perceptual depth of S3D videos during shooting, even for professionals with years of experience [8]. Existing S3D video post-processing technology [9, 10] helps to manipulate the original disparity of S3D images and videos. Given the desired disparity mapping, these methods manipulate the original disparity to meet the requirements. Unfortunately, such approaches require manually input disparity targets or manipulation operators for guidance. A general, content-driven solution for ensuring the comfort of S3D video is still lacking.

In this paper, we provide an automatic solution to the disparity tuning problem using a unified 


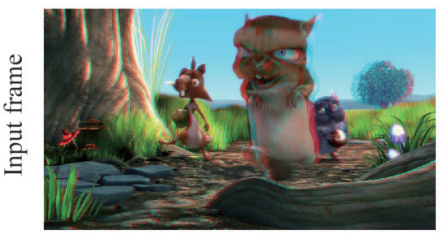

(a)

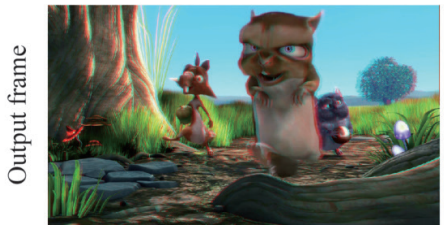

(e)

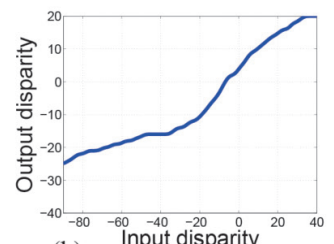

(b)

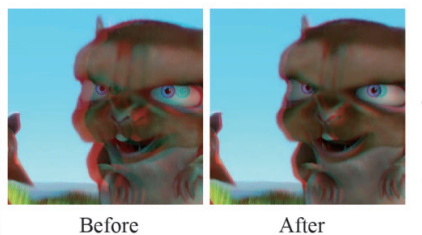

(f)

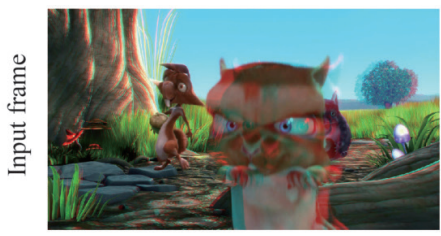

(c)

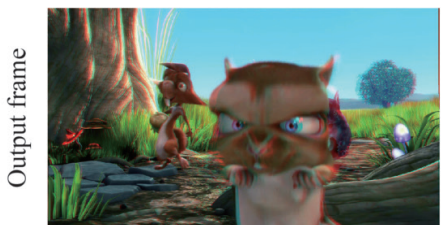

(g)

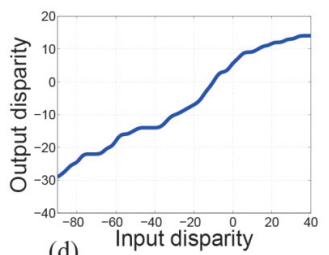

(d)

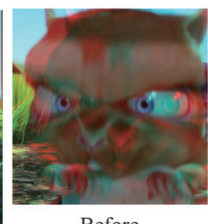

Before

(h)

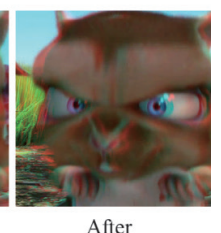

)

Fig. 1 Inputs and outputs: given an input stereoscopic 3D video (sample frames (a) and (c)), our framework automatically determines comfort-driven disparity mappings (b) and (d) for every frame. Output video frames (e) and (g) are produced by applying these mappings to the input video frames, improving visual comfort. (f) and (h) show close-ups of frames before and after manipulation (c) Blender Foundation).

and extensible comfort-driven framework. Unlike previous works that focus on user-guided S3D video disparity retargeting $[9,10]$, we automatically manipulate the disparity of an original S3D video, to improve visual comfort while maintaining satisfactory parts of the original content whenever possible. The challenge of this problem is to build a bridge between S3D visual comfort and the automatic manipulation of video content. By taking advantage of existing S3D visual comfort models, we derive a general discomfort metric which we use to evaluate and predict the discomfort level. We build on this metric to define an objective function for use in optimising disparity mapping functions. Our metric may be further extended if needed, to incorporate further visual comfort models. We optimise the mappings over the whole video, using a hierarchical solution based on a genetic algorithm. The output video is generated by applying the disparity mappings to the original video using a warping-based technology (Fig. 1). To our knowledge, our framework is the first system which can automatically improve visual comfort by means of comfort-driven disparity adjustment.

The major contributions of our work are thus:

- A unified S3D video post-processing framework that automatically reduces visual discomfort by disparity adjustment.

- A discomfort metric that combines several key visual comfort models; it could be easily extended to incorporate others too if desired. It provides a basis for an objective function used to optimise disparity.
- A hierarchical optimisation method for computing a disparity mapping for each video frame.

\section{Related work}

Causes of visual discomfort experienced when watching S3D movies have been investigated, with a view to improving such movies. Mendiburu [8] qualitatively determined various factors such as excessive depth and discontinuous depth changes that contribute to visual fatigue. Liu et al. [11] summarized several principles, and applied them to photo slideshows and video stabilization.

Various mathematical models have also been proposed to quantitatively evaluate discomfort experienced by the HVS. Besides viewing configurations such as viewing distance [12], time [13], and display screen type, effects particular to stereoscopic content have also been widely investigated [3, 4, 14-18], which we now consider in turn.

Vergence-accommodation conflict is widely accepted to be a key factor in visual discomfort. These ideas may be used to quantitatively determine a comfort zone within which little discomfort arises [12]. Stereoscopic fusion disparity range is modeled in Ref. [19], based on viewing distance and display sizes. Didyk et al. [3] modeled perceptual disparity based on experiments with sinusoidal stimuli; the ideas can be used to produce backwardcompatible stereo and personalized stereo. This work was later extended to incorporate the influence of luminance contrast [4]. Our metric includes a 
disparity range term, based on the comfort zone model in Ref. [12]. It allows us to decide whether the disparity of a given point lies within the comfort zone.

Motion is another important factor in perceptual discomfort [14, 15, 20]. In Ref. [20], the contribution of the velocity of moving objects to visual discomfort is considered. Jung et al. [15] gave a visual comfort metric based on salient object motion. Cho and Kang [13] conducted experiments with various combinations of disparity, viewing time, and motionin-depth, measuring the visual discomfort. Du et al. [14] proposed a comfort metric for motion which takes into account the interaction of motion components in multiple directions, and depths. Such literature shows that visual comfort is improved when objects move at lower velocities or lie closer to the screen plane. Movements perpendicular to the screen (along the $z$-axis) play a more powerful role in comfort than movements parallel to the screen plane (the $x-y$ plane).

Abrupt depth changes at scene discontinuities may also induce discomfort: for instance, a sudden jump from a shot focused in the distance to an extreme close-up can be disorienting. Disparity-response time models $[17,18]$ have been determined by a series of user-experience experiments. To reduce discomfort caused by depth changes, depths in shots should change smoothly.

Stereoscopic window violation describes a situation in which any object with negative disparity (in front of the screen) touches the left or right screen boundary. Part of the object may be seen by one eye but hidden from the other eye, leading to confusion by the viewer as to the object's actual position; this too causes fatigue [21]. Yet further factors are discussed in a recent survey [22]. As our approach provides a post-processing tool, we consider factors related to scene layout rather than camera parameters. These factors are disparity range, motion, stereoscopic window violation, and depth continuity; they are meant to cover the major causes of discomfort, but our approach could be extended to include others too.

Use of post-processing technology has increased in recent years, helping amateurs to create S3D content and directors to improve S3D movie appearance. Lo et al. [23] showed how to perform copy \& paste for S3D, to create new stereoscopic photos from old ones; constraints must be carefully chosen. Later, Tong et al. [24] extended this work to allow pasting of 2D images into stereoscopic images. Kim et al. [25] provided a method to create S3D line drawings from 3D shapes. Niu et al. [26] gave a warping-based method for stereoscopic image retargeting. Lang et al. [10] provided a disparity manipulation framework which applies desired disparity mapping operators to the original video using image warping. Kellnhofer et al. [9] optimised the depth trajectories of objects in an S3D video, providing smoother motion. Kim et al. [27] computed multi-perspective stereoscopic images from a light field, meeting users' artistic control requirements. Masia et al. [28] proposed a light field retargeting method that preserves perceptual depth on a variety of display types. Koppal et al. [29] provided an editor for interactively tuning camera and viewing parameters. Manually tuned parameters of cameras are applied to video; the results are then immediately fed back to the user. However, there is presently a gap between mathematical comfort models and post-processing applications - few technologies automatically work in a comfort-driven manner.

In a similar vein to our work, the OSCAM system [30] automatically optimises the camera convergence and interaxial separation to ensure that $3 \mathrm{D}$ scene contents are within a comfortable depth range. However this work is limited to processing virtual scenes with known camera settings. Tseng et al. [31] automatically optimised parameters of S3D cameras, taking into account the depth range and stereoscopic window violation. The major differences between their work and ours are, firstly, they optimise the camera separation and convergence, while our system automatically generates an output video with a better viewing experience. Secondly, their objective functions are derived from either a simple depth range or a few general principles while ours relys on mathematical models. We build upon existing S3D post-processing approaches, especially warping-based ones, to build a bridge between comfort models and a practical tool.

\section{Overview}

In this section, we explain our notation, and then sketch our proposed framework. 
We adapt the measure of binocular disparity from Ref. [14], expressed as angular disparity. Assuming the viewer focuses on the display screen with a vergence angle $\theta^{\prime}$, the angular disparity at a $3 \mathrm{D}$ point $P$ with a vergence angle $\theta$ is measured as the difference of vergence angles $\theta^{\prime}-\theta$ (see Fig. 2(a)). We also use the concept of pixel disparity in the metric and disparity mapping optimisation. The pixel disparity of a feature point $f_{\mathrm{L}}$ in the left view $\mathrm{L}$ is defined as an integer offset $f_{\mathrm{R}}-f_{\mathrm{L}}$ where $f_{\mathrm{R}}$ is the corresponding feature location in the right view $\mathrm{R}$ (see Fig. 2(b)). Given these definitions, both the angular disparity and pixel disparity are negative when the $3 \mathrm{D}$ point $P$ is in front of the screen and positive when it is behind the screen. A disparity mapping is a function $\phi(d)$ that given an input disparity value $d$, returns a new output disparity value $d^{\prime}$. In this paper, $\phi$ is presented in discrete form: given a set of $\tau$ different disparity values $D_{\text {in }}=\left\{d_{\min }, \ldots, d_{\tau}\right\}$, and a corresponding set of output disparity values $D_{\text {out }}=\left\{d_{\min }^{\prime}, \ldots, d_{\tau}^{\prime}\right\}$, we regard $\phi: D_{\text {in }} \rightarrow D_{\text {out }}$ as a disparity mapping, where $d_{i}^{\prime}=\phi\left(d_{i}\right)$.

As explained in Section 1, our comfort-driven disparity mapping framework automatically adjusts the disparity in an S3D video to improve visual comfort. Given an input S3D video to be optimised, we first evaluate the discomfort level of every

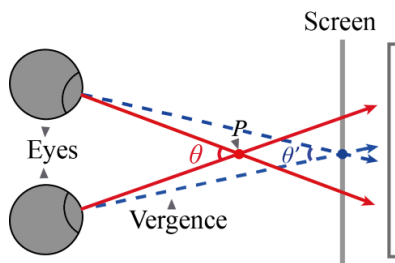

(a)

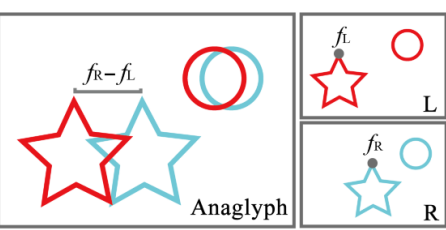

(b)
Fig. 2 Definitions: (a) angular disparity and (b) pixel disparity. frame, using the proposed metric, then determine time intervals which cause discomfort and key frames inside each interval throughout the video (see Section 4). Next, based on the key frames, we optimise a disparity mapping $\phi$ for every frame using a hierarchical optimisation method (see Section 5), using an objective function derived from the discomfort metric. Finally, the mappings are applied to the original video by video warping. The pipeline is illustrated in Fig. 3.

\section{Discomfort metric}

An objective function measuring discomfort level is essential for automatic S3D video comfort optimisation. In this section, we present a general discomfort metric which is used to determine the objective function for disparity mapping optimisation. The metric takes into account disparity range, motion, stereoscopic window violation, and temporal smoothness, all of which have been shown to have a major impact on the HVS. Each factor is formulated as a cost function. The temporal smoothness term relates pairs of successive frames (so is a binary term) while others are only dependent on one frame (so are unary terms). The wide-ranging nature of this metric enables us to evaluate discomfort level in the round. The disparity range term measures the severity of vergence-accommodation conflict. The motion term evaluates discomfort brought about by eye movements. Retinal rivalry arises from inconsistent screen boundary occlusions, and is assessed by the stereoscopic window violation term. Flickering resulting from temporal inconsistency is evaluated by the temporal smoothness term. We now discuss each term individually and then explain how they

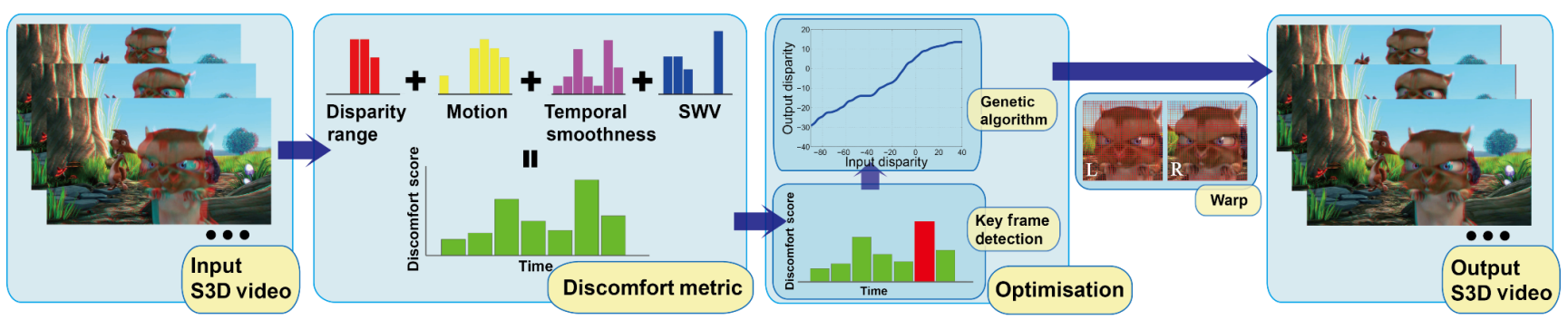

Fig. 3 Pipeline. The input of our system is a stereoscopic 3D video. Discomfort level of every frame is evaluated using the proposed metric. Discomfort intervals and key frames inside each interval are determined. A disparity mapping for every frame is optimised, based on the key frames, using a hierarchical optimisation method. Finally, the output video is generated by applying the mappings to the original video by warping. 
are combined.

\subsection{Individual terms}

Disparity range. Excessive disparity leads to strong adverse reactions in the HVS due to vergenceaccommodation conflict $[5,6]$. To reduce the resulting discomfort, one intuitive approach is to compress the disparity range, but severe compression makes an S3D video appear flat, and ultimately imperceptibly different from 2D. Instead, we evaluate how far each $3 \mathrm{D}$ point is from the screen plane and penalize points that fall outside the comfort zone. In Ref. [12], the far and near comfort zone boundaries $B_{\text {far }}$ and $B_{\text {near }}$ are introduced. In terms of angular disparity, these may be written as

$$
\begin{aligned}
B_{\mathrm{far}}= & 2 \tan ^{-1}\left[\frac{d_{\mathrm{a}}}{2 m_{\mathrm{far}} d_{\mathrm{f}}}\left(1-T_{\mathrm{far}} d_{\mathrm{f}}\right)\right] \\
& -2 \tan ^{-1}\left(\frac{d_{\mathrm{a}}}{2 d_{\mathrm{f}}}\right) \\
B_{\text {near }}= & 2 \tan ^{-1}\left[\frac{d_{\mathrm{a}}}{2 m_{\text {near }} d_{\mathrm{f}}}\left(1-T_{\text {near }} d_{\mathrm{f}}\right)\right] \\
& -2 \tan ^{-1}\left(\frac{d_{\mathrm{a}}}{2 d_{\mathrm{f}}}\right)
\end{aligned}
$$

where the constants in their model are $m_{\text {far }}=1.129$, $m_{\text {near }}=1.035, T_{\text {far }}=0.442$, and $T_{\text {near }}=-0.626$. $d_{\mathrm{a}}$ is the angular disparity (in degree) of a pixel and $d_{\mathrm{f}}$ is the viewing distance (in metre), which, in our viewing configuration, is set to $0.55 \mathrm{~m}$.

In this formulation, whether the angular disparity $d_{\mathrm{a}}(p)$ of a pixel $p$ is within the comfort zone range is determined by

$$
\delta(p)= \begin{cases}1, & \text { if } B_{\text {near }}<d_{\mathrm{a}}(p)<B_{\text {far }} \\ 0, & \text { otherwise }\end{cases}
$$

The fraction of pixels in frame $f$ whose disparity is outside the comfort zone is computed, and used to define the disparity range penalty term $E_{\mathrm{d}}(f)$ for frame $f$ :

$$
E_{\mathrm{d}}(f)=1-\frac{1}{N} \sum_{p \in f} \delta(p)
$$

where $N$ is the number of pixels in frame $f$. Figure 4 shows examples where disparities of certain pixels lie beyond the comfort zone.

Motion is an important source of visual discomfort [15, 20]. In Ref. [14], a novel visual comfort metric for S3D motion is proposed. This metric is a function of both the combination of velocity and depth, and luminance frequency. It returns a comfort value from 1 to 5 (the higher,

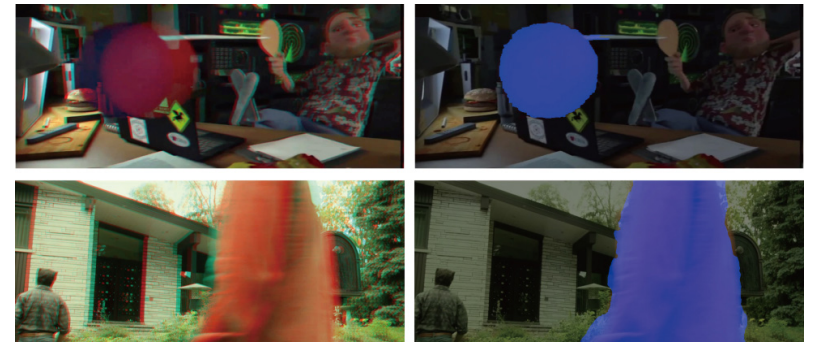

Fig. 4 Comfort zone. Left: anaglyph 3D images. Right: disparities beyond the comfort zone shown in blue.

the more comfortable). We adopt this model in our metric and assign to every video frame a motion discomfort value. We first assign a motion discomfort value $V_{\mathrm{c}}(p)=\omega_{\mathrm{n}}\left(5-M_{\mathrm{p}}(p)\right)$ for every pixel $p$, where $\omega_{\mathrm{n}}$ is a coefficient normalising $V_{\mathrm{c}}(p)$ to $[0,1)$, set to $0.25 . M_{\mathrm{p}}(p)$ is the pixel-wise motion comfort value calculated as in Ref. [14]:

$$
M_{\mathrm{p}}(p)=\sum_{k=0}^{n} C\left(v_{x y}, v_{z}, d, \frac{f_{l_{0}}}{2^{k}}\right) \times \frac{L_{k}(p)}{\sum_{k} L_{k}(p)}
$$

where $C\left(v_{x y}, v_{z}, d, \frac{f_{l_{0}}}{2^{k}}\right)$ is a model of motion comfort based on planar velocity $v_{x y}$, spatial velocity $v_{z}$, angular disparity $d$, and luminance frequency $\frac{f_{l_{0}}}{2^{k}}$. $L_{k}(p)$ is the contrast value of the $\left(2^{k+1}+1\right)$ neighborhood at $p$ at the $k$-th Laplacian level of the Laplacian pyramid of the luminance; see Ref. [14] for further details.

After computing a discomfort value for every pixel, we determine the motion discomfort for the whole frame. In Ref. [14], average motion comfort values are calculated for individual saliency-based segments [32], assigning an importance value to every segment. The segments are obtained by graph-based segmentation [33]. They assumed that the most uncomfortable region in a frame dictates the discomfort of the whole frame. However, we find that calculating the most salient and uncomfortable region in separate images without considering temporal coherence can lead to motion comfort instability. Instead, we modify their approach to perform SLIC superpixel segmentation [34], consider multiple discomfort-causing segments, and regard every segment as having the same importance. We extract an average motion comfort value for the top- $K$ ( $K=20$ by default) segment discomfort values as a motion penalty. The motion discomfort $E_{\mathrm{m}}(f)$ 
for the whole frame $f$ is

$$
E_{\mathrm{m}}(f)=\frac{1}{K} \sum_{k=1}^{K} T\left(V_{\mathrm{s}}\left(s_{k}\right)\right)
$$

where $V_{\mathrm{s}}(s)=\sum_{p \in s} V_{\mathrm{c}}(p) / m$ is the average motion discomfort value for a segment $s$ having $m$ pixels. $T(\cdot)$ is the set of segment motion discomfort values, in descending order, as computed in Ref. [14]. Figure 5 shows example S3D frames with segmentwise discomfort maps according to motion.

Stereoscopic window violation occurs when an object is virtually located in front of the screen (i.e., has negative disparity) but is occluded by the screen boundary. This is confusing as a nearer object appears to be occluded by a further one, causing retinal rivalry [8]. If this happens, only one eye can see part of the object, leading to visual inconsistency and hence discomfort (see Fig. 6). One practical way to alleviate this is to trim off the offending part.

To measure stereoscopic window violation (SWV), we use a term $E_{\mathrm{v}}(f)$ for frame $f$. We first detect violations by checking pixels near left and right boundaries: if pixels touching the frame boundaries have negative disparity, they violate the stereoscopic

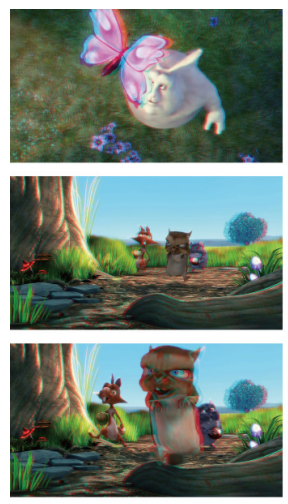

(a)

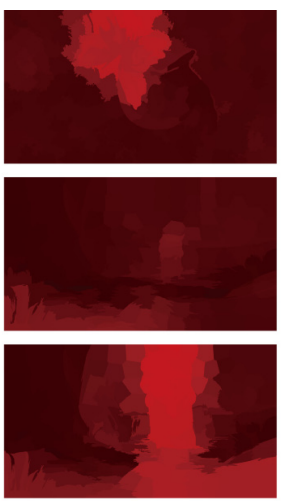

(b)
Uncomfortable

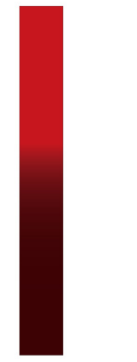

Comfortable
Fig. 5 Motion discomfort estimation: (a) anaglyph 3D frames (C) Blender Foundation); (b) estimated discomfort caused by motion.
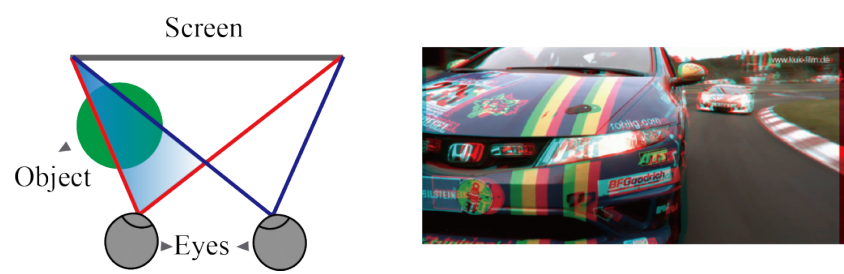

Fig. 6 Stereoscopic window violation (SWV). Left: a toy example illustrating SWV. Part of the object in green falling in the light blue region can only be seen by the left eye. Right: a real S3D photo showing SWV (C) KUK Filmproduktion $\mathrm{GmbH}$ ). There is inconsistent content in the leftmost part of the photo, leading to viewer discomfort. window. The SWV penalty for frame $f$ is then defined by counting the number of pixels included in violating objects:

$$
E_{\mathrm{v}}(f)=\frac{1}{N} \sum_{s \in R_{\mathrm{b}}} n(s)
$$

where $s$ stands for image segments extracted as before, and $R_{\mathrm{b}}$ is an approximation of violating objects in the form of segments; every segment in $R_{\mathrm{b}}$ has a negative average disparity. $R_{\mathrm{b}}$ is initially set to those boundary segments with a negative average disparity, and is then iteratively augmented by adding new neighbouring segments with negative average disparity until no new segments with negative average disparity are found. $n(s)$ is the number of pixels in segment $s$ and $N$ is the number of pixels in frame $f$.

Temporal smoothness. To avoid sudden depth changes, the disparity should vary smoothly and slowly, as needed. In Ref. [11], the importance of temporal smoothness is emphasised in 3D cinematography; they suggested that the disparity range of successive frames should vary smoothly. Following the definition of disparity map similarity in Ref. [11], we define the similarity of disparity between neighbouring frames $f$ and $f^{\prime}$ using JensenShannon divergence [35]:

$E_{\mathrm{S}}\left(f, f^{\prime}\right)=H\left(\frac{\Psi(f)+\Psi\left(f^{\prime}\right)}{2}\right)-\frac{H(\Psi(f))+H\left(\Psi\left(f^{\prime}\right)\right)}{2}$

where $\Psi(f)$ is a pixel disparity histogram for frame $f$ with $d_{\max }-d_{\min }+1$ bins; $d_{\max }$ is the largest integer pixel disparity value in $f$ and $d_{\text {min }}$ is the smallest integer pixel disparity value in $f . H(X)$ is the Shannon entropy for distribution $X$. Intuitively, the more unlike the disparity histograms are, the higher the value of $E_{\mathrm{s}}$.

\subsection{Discomfort metric}

Our general discomfort metric for a set of successive frames $\widehat{F}$ in an S3D video is formulated as a linear combination of the above terms in Eqs. (3)-(6), summed over the frames:

$$
\left.\begin{array}{rl}
E_{\mathrm{c}}(\widehat{F})= & \sum_{f \in \widehat{F}} E_{\mathrm{c}}(f) \\
E_{\mathrm{c}}(f)= & \lambda_{\mathrm{d}} E_{\mathrm{d}}(f)+\lambda_{\mathrm{m}} E_{\mathrm{m}}(f) \\
& +\lambda_{\mathrm{v}} E_{\mathrm{v}}(f)+\lambda_{\mathrm{s}} E_{\mathrm{s}}\left(f, f^{\prime}\right)
\end{array}\right\}
$$

where $f^{\prime}$ is the successor frame to $f$ in $\widehat{F} . \lambda_{\mathrm{d}}, \lambda_{\mathrm{m}}$, $\lambda_{\mathrm{v}}$, and $\lambda_{\mathrm{s}}$ are weights balancing the penalty terms, 
set to $1,0.4,10$, and 0.1 respectively. The weights are determined via experiments. We did a small scale perceptual study on 10 subjects with 10 input videos: we enumerated every weight from 0 to 20 with step 0.1 , generating $1.6 \times 10^{9}$ possible combinations of weights. After calculating the corresponding general metrics for input videos based on each group of weights, we asked 5 subjects to view each video and evaluate its comfort level by assigning integer scores from 1 to 5 . We finally selected the group of weights, for which the metric score best reflected the subjects' comfort feelings. The weights were further validated by the other 5 subjects' evaluation. This metric can be used to predict the overall discomfort level of part or all of an S3D video. An S3D video frame is predicted as visually comfortable if the discomfort value $E_{\mathrm{c}}<0.3$. Figure $7(\mathrm{~b})$ shows example frames with their corresponding discomfort values.

The metric has a general form, with a default set of weights balancing the penalty terms. If considered unimportant, certain terms can be ignored, by setting their corresponding weights to 0. Alternatively, additional terms of a similar kind could also be included with proper weight configuration (as an example, we present a variation of the metric, driving perceptual depth enhancement, by adding another unary term to each frame in the video (see Section 6)). We intentionally do not include all factors that cause visual fatigue there are many such factors. Instead, we claim that the above metric includes many of the most significant factors, and the way we have formulated it allows ready extension to include other comfort

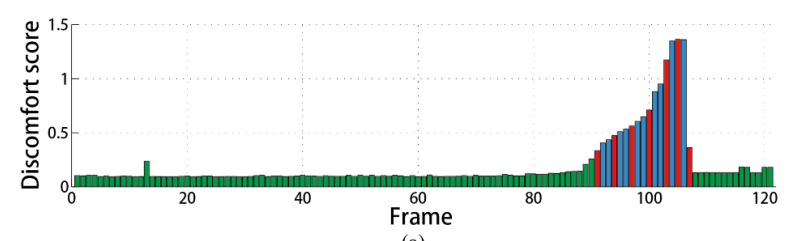

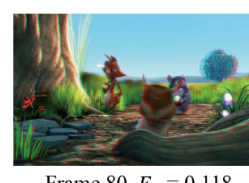

Frame $80, E_{\mathrm{C}}=0.118$

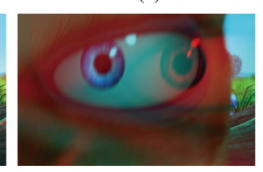

Frame $105, E_{\mathrm{c}}=1.364$ (b)

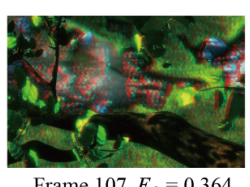

Frame $107, E_{\mathrm{c}}=0.364$
Fig. 7 Typical frames (c) Blender Foundation) and discomfort scores. (a) Discomfort scores for frames in an S3D video clip. The discomfort interval is marked in blue. Key frames selected by our algorithm are highlighted in red. (b) shows three frames and corresponding discomfort scores from (a). models using additional penalty terms. The ideas in the rest of the paper do not depend on the precise form of this metric, only that such a metric can be formulated. We next show how to use this metric to define the objective function used to optimise pixel disparity mapping.

\section{Optimisation of pixel disparity mapping}

Based on the above visual discomfort metric, we next derive the objective function used for disparity mapping optimisation. A genetic algorithm is used in a hierarchical approach to optimise disparity mapping: given a set of input disparity values, we compute a corresponding target output disparity for each value.

\subsection{Objective function}

The visual discomfort metric $E_{\mathrm{c}}$ measures the discomfort level of S3D video frames. However, directly using it as an objective function in an optimisation process leads to unsatisfactory results: clearly, mapping all disparity values to zero would minimise $E_{\mathrm{c}}$, making it equal to zero at all times. Also, making large changes to the disparity without scaling the sizes of objects leads to a change in the perceived size of the original content. We thus add an additional unary term $E_{\mathrm{n}}(\phi, f)$ to every frame $f$ with the intent that optimisation should change the original disparities as little as possible. $E_{\mathrm{n}}(\phi, f)$ measures differences between new and original disparities:

$$
E_{\mathrm{n}}(\phi, f)=\frac{1}{N} \sum_{d \in\left[d_{\min }, d_{\max }\right]} \Psi_{d}(f)|\phi(d, f)-d|
$$

where $N$ is the number of pixels in frame $f, d$ is the integer pixel disparity value, and $\Psi_{d}(f)$ is the disparity histogram count for disparity $d$ in frame $f$, as in Eq. (6). $\phi(d, f)$ is disparity mapping for disparity $d$ in frame $f$. This formulation gives a cost for the mapping $\phi$, punishing large changes from the original disparity distribution. This additional term allows us to find a suitable disparity mapping for each video frame that improves visual comfort while also preserving the original appearance.

We denote the objective function for optimising a sequence of mappings $\widehat{\Phi}$ of a sequence of frames $\widehat{F}$ in a $\mathrm{S} 3 \mathrm{D}$ video as $E(\widehat{\Phi})$; it is defined as 


$$
\begin{aligned}
E(\widehat{\Phi})= & \sum_{f \in \widehat{F}}\left(\lambda_{\mathrm{d}} E_{\mathrm{d}}\left(\Gamma_{\phi_{f}}(f)\right)+\lambda_{\mathrm{m}} E_{\mathrm{m}}\left(\Gamma_{\phi_{f}}(f)\right)\right. \\
& +\lambda_{\mathrm{v}} E_{\mathrm{v}}\left(\Gamma_{\phi_{f}}(f)\right)+\lambda_{\mathrm{n}} E_{\mathrm{n}}\left(\phi_{f}, f\right) \\
& \left.+\lambda_{\mathrm{s}} E_{\mathrm{s}}\left(\Gamma_{\phi_{f}}(f), \Gamma_{\phi_{f^{\prime}}}\left(f^{\prime}\right)\right)\right)
\end{aligned}
$$

where $f^{\prime}$ is the successor frame to $f$, and $\Gamma_{\phi_{f}}(f)$ is a function which applies the mapping operator $\phi_{f}$ to frame $f$ to produce a new frame with the desired new pixel disparities. $\lambda_{\mathrm{n}}$ is a further weight, set to 0.01 by default.

\subsection{Hierarchical optimisation}

The objective function in Eq. (9) is complex; we use an efficient hierarchical approach to optimise it in a coarse-to-fine manner along the time-line. We note that in S3D movies, frames causing discomfort usually appear together, forming discomfort intervals. Thus, we firstly extract discomfort intervals for the whole video: we manipulate the disparity only for frames which cause discomfort, and leave the others unchanged. The discomfort intervals are determined using Eq. (7): a discomfort interval is a set of continuous frames from starting frame $f_{\mathrm{s}}$ to ending frame $f_{\mathrm{e}}$, within which the discomfort metric $E_{\mathrm{c}}\left(\left\{f, f^{\prime}\right\}\right)$ is above a threshold $\alpha=0.3$, where $f$ and $f^{\prime}$ are consecutive frames inside the interval. During optimisation, at coarser levels, inside every discomfort interval we determine key frames where the disparity changes drastically or there is a local maximum of discomfort. Frames at discomfort interval boundaries are also taken as key frames having a fixed identity pixel disparity map $(\phi(d)=d)$. Next, we use a genetic algorithm to optimise pixel disparity mappings of the key frames, treating the key frames as neighbours. After optimising the key frames at this hierarchy level, we fix the disparity mappings of the current key frames, and continue to seek new key frames for finer intervals between any two successive key frames at the current level. The mappings of the current key frames are used as boundary conditions for the next level. This process is recursively performed until fewer than ten frames exist between each neighbouring pair of key frames. Finally, the disparity mapping for remaining frames between these key frames is interpolated. We now give further details of key steps.

\subsubsection{Key frame determination}

Key frame determination is a crucial step in the hierarchical disparity mapping optimisation. Since the optimisation is performed in a coarse-to-fine manner, at coarser levels, key frames should provide a story line overview of frames at finer levels, especially in terms of disparity. Motivated by this requirement, inside each discomfort interval we mark a frame as a key frame when there is a sudden depth change or the discomfort metric reaches a local maximum within a window of $\Upsilon_{l}$ frames for each level $l$. By default, $\Upsilon_{l}$ at level $l$ is set to a quarter of the interval length between the two boundary key frames. Specifically, we use the inequality $E_{\mathrm{s}}\left(f, f^{\prime}\right)>\beta$ to determine whether frame $f$ is a key frame at a drastic depth change; by default $\beta=0.5$. After optimising key frames at level $l$, new key frames at level $l+1$ are recursively determined, by seeking new key frames at level $l+1$ between every adjacent pair of key frames at level $l$. We stop when fewer than ten frames exist between each neighbouring pair of key frames.

\subsubsection{Heuristic optimisation using genetic algorithm}

After finding key frame sets $F$ at level $l$, we use a heuristic algorithm to optimise disparity mappings of these key frames. Without loss of generality, assume we are optimising a discomfort interval with $t$ detected key frames $F=\left\{f_{1}, \ldots, f_{t}\right\}$. Including the additional key frames at the discomfort interval boundaries, the augmented key frame set becomes $\underline{F}=\left\{f_{\mathrm{s}}, f_{1}, \ldots, f_{t}, f_{\mathrm{e}}\right\}$, with fixed identity disparity mappings for $f_{\mathrm{s}}$ and $f_{\mathrm{e}}$ as boundary conditions. We regard every successive pair of frames in $\underline{F}$ along the time-line as neighbours in a coarse view. We optimise the key frame mappings $\Phi=\left\{\phi_{1}, \ldots, \phi_{t}\right\}$ at coarser levels using the objective function adapted from Eq. (9):

$$
\begin{aligned}
E(\Phi)= & \sum_{f \in F}\left(\lambda_{\mathrm{d}} E_{\mathrm{d}}\left(\Gamma_{\phi_{f}}(f)\right)+\lambda_{\mathrm{m}} E_{\mathrm{m}}\left(\Gamma_{\phi_{f}}(f)\right)\right. \\
& +\lambda_{\mathrm{v}} E_{\mathrm{v}}\left(\Gamma_{\phi_{f}}(f)\right)+\lambda_{\mathrm{n}} E_{\mathrm{n}}\left(\phi_{f}, f\right) \\
& \left.+\lambda_{\mathrm{s}} E_{\mathrm{s}}\left(\Gamma_{\phi_{f}}(f), \Gamma_{\phi_{f^{\prime}}}\left(f^{\prime}\right)\right)\right)
\end{aligned}
$$

where $f^{\prime}$ is the successor frame to $f$ in $\underline{F}$. This objective function is used as fitness assessment in genetic algorithm.

A genetic algorithm (GA) is used to optimise the disparity mapping $\phi$ for each frame $f$ using this objective function as a fitness function. We use the GALib implementation of a steady-state genetic 
algorithm [36]; $50 \%$ of the population is replaced on each generation. The genome for each individual is a vector of real numbers, which is used to store target disparity mapping values (with a conversion between integer and real numbers). Uniform crossover [37] is used with Gaussian mutation [38], which adds a random value from a Gaussian distribution to each element of an individual's state vector, to create offspring.

Genome of individuals. The genome representation needs to be carefully designed; poor choice can lead to GA divergence. The target output disparity mapping values $D_{\phi_{f}}=$ $\left\{\phi\left(d_{\min }\right), \ldots, \phi\left(d_{\max }\right)\right\}$ of the mapping function $\phi$ for a frame $f$ is an elementary unit in each individual's genome. The disparity mapping $\phi(x)$ should be a non-decreasing function, i.e., if $x_{1}<x_{2}$, then $\phi\left(x_{1}\right) \leqslant \phi\left(x_{2}\right)$, to avoid depth reversal artifacts in the output video. We enforce this requirement by using an increment-based representation. We represent the mapping values $D_{\phi_{f}}=\left\{\phi\left(d_{\text {min }}\right)\right.$, $\left.\ldots, \phi\left(d_{\max }\right)\right\}$ as $\widetilde{D_{\phi_{f}}}=\left\{\phi\left(d_{\min }\right), \Delta_{1}, \ldots, \Delta_{p-1}\right\}$ where $d$ ranges over all integer pixel disparity values between $d_{\min }$ and $d_{\max }$, and $\Delta_{i}=\phi\left(d_{i+1}\right)-\phi\left(d_{i}\right)$ is a non-negative mapping value increment. Obviously, we can recover $D_{\phi_{f}}$ from the relationship $D_{\phi_{f}}[i]=$ $\widetilde{D_{\phi_{f}}}[i-1]+\widetilde{D_{\phi_{f}}}[i]$. The non-negativity of $\Delta_{i}$ is guaranteed by additional bound $\overline{b_{i}}$ and lower bound $\underline{b_{i}}$ on each integer element of $\widetilde{D_{\phi_{f}}}$ :

$$
\overline{b_{i}}= \begin{cases}d_{\min }+\left(d_{\max }-d_{\min }\right) / 2, & \text { if } i=1 \\ 5, & \text { otherwise }\end{cases}
$$

and

$$
\underline{b_{i}}= \begin{cases}d_{\min }-\left(d_{\max }-d_{\min }\right) / 2, & \text { if } i=1 \\ 0, & \text { otherwise }\end{cases}
$$

These upper and lower bounds also prevent the mappings from making over-large increments $\Delta_{i}$. This constraint is supported by the steady-state genetic algorithm. The full genome of each individual is a vector of integers which concatenates the mapping values $\widetilde{D_{\Phi}}=\left\{\widetilde{D_{\phi_{1}}}, \ldots, \widetilde{D_{\phi_{t}}}\right\}$ for the $t$ key frames in $F$.

Evolution. The state of every individual in the first generation is initialised using random mappings. The objective function in Eq. (10) is used for individual fitness assessment. The uniform crossover probability is $p_{\mathrm{c}}=0.7$ and the Gaussian mutation probability is $p_{\mathrm{m}}=0.05$. The population size $n_{\mathrm{p}}$ is set to 100 and the GA terminates after $n_{\mathrm{g}}=$ 50 generations. As a steady-state GA is used, the last generation includes the best solution found for the desired mappings $\Phi^{\prime}$. Figure 8 illustrates the mappings corresponding to the best solution in different generations.

\subsection{Warping-based manipulation}

After optimising pixel disparity mappings for each frame of the video, we have to adjust the input video using these mappings. In Ref. [10], a warping-based method is given to adjust disparity to match desired disparity mappings. Their approach first extracts sparse stereo correspondences, followed by warping of left and right frames respectively with constraints applied to the vertices of a mesh grid placed over each frame. The output is thus a deformed mesh as
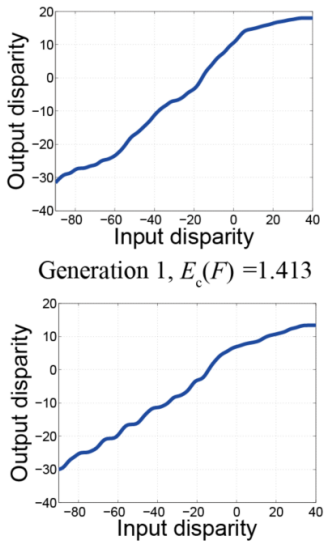

Generation $13, E_{\mathrm{c}}(F)=1.189$
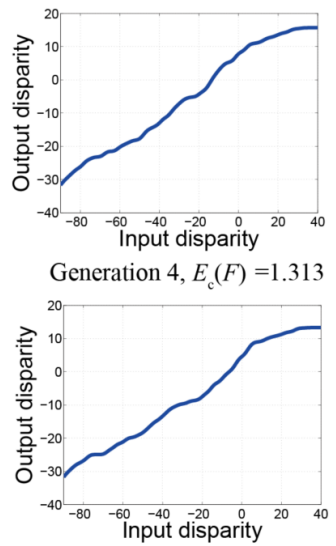

Generation 22, $E_{\mathrm{c}}(F)=1.172$
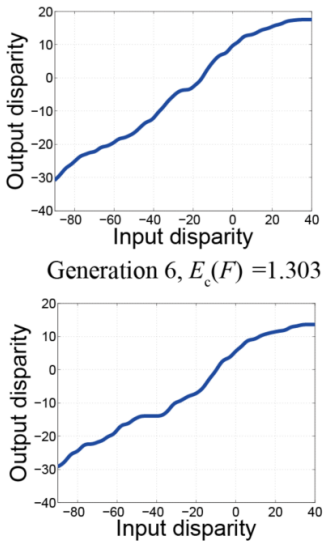

Generation 35, $E_{c}(F)=1.150$
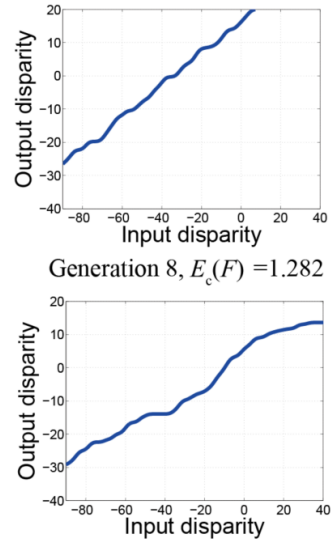

Generation $45, E_{\mathrm{c}}(F)=1.150$
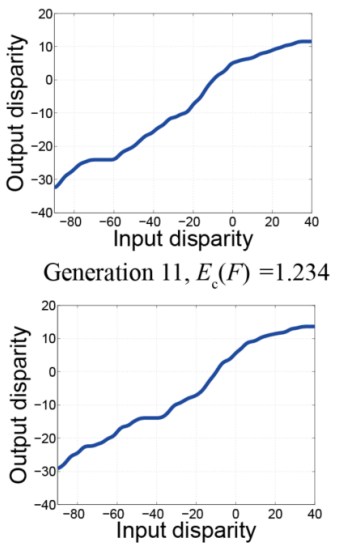

Generation $50, E(F)=1.150$

Fig. 8 Best disparity mapping solutions for improving comfort level of the frame shown in Fig. 1(c), at various generations of the genetic algorithm. The frame set $F$ contains four key frames. During optimisation, the discomfort cost $E_{\mathrm{c}}$ of $F$ is reduced. 
well as the warped frame. We use this technology to generate the output video.

\section{Results}

The experiments were carried out on a computer with an Intel Core i7-4790K CPU with 32 GB RAM. All videos were uniformly scaled to fit the screen size $(1920 \times 1080$ pixels $)$ to the extent possible before calculation. We calculate dense pixel correspondence between the left view and right view to estimate the pixel disparity in S3D videos using optical flow [39]. Motion in the $x-y$ plane is also estimated using this method, between consecutive frames in the left view. Calculating the discomfort metric for one S3D video frame of size $1920 \times 1080$ takes less than $0.2 \mathrm{~s}$. The most time-consuming part is hierarchical optimisation, but the time taken is variable. It is dominated by the key frame determination step; it takes up to $15 \mathrm{~min}$ to optimise ten key frames together in our implementation, using a single core.

We have tested our approach on S3D video clips whose lengths are less than one minute. As explained in Ref. [14], the proposed motion comfort metric was derived from experiments on short videos. All of the results were obtained using default parameters. Extensive experiments showed that our system is insensitive to parameter settings.

Our method provides smooth scene transitions between successive shots. Representative frames of a video clip with shot cuts are shown in Fig. 9(a).
Boundary frames 1 and 40 do not cause discomfort, so are fixed to retain their original disparities. Our algorithm detects drastic disparity changes between these boundary frames and automatically adjusts disparities to provide smoother depth transitions by finding suitable disparity mappings (see Fig. 9(b)). In this example, frames where shot cuts occur are detected as key frames. This is because the values of motion term and temporal smoothness term reach a local maximum within a window. As can be seen in Fig. 9(c), after manipulating the video, the depth storyboard suffers less from sudden jumps in disparity. While the last part of the video initially has a constant disparity range, which after processing becomes a slowly increasing disparity range, this does not lead to any perceptual artifacts: (i) slow transitions in disparity are often used to control disparity at shot cuts, (ii) the rate of disparity change is small, and (iii) the warping provides a smooth solution.

Figure 10 gives an example of automatic correction of excessive disparity range. The ball popping out towards the viewer in the center of the frame makes it difficult for the viewer to comfortably perceive the depth. Our correction pushes the ball a little closer to the screen. Pushing the ball back into the screen too far would change the content too much, in disagreement with the film maker's intent. The deformed meshes of the left and right views used for the warping-based disparity manipulation are also shown. Discomfort scores in our metric before and

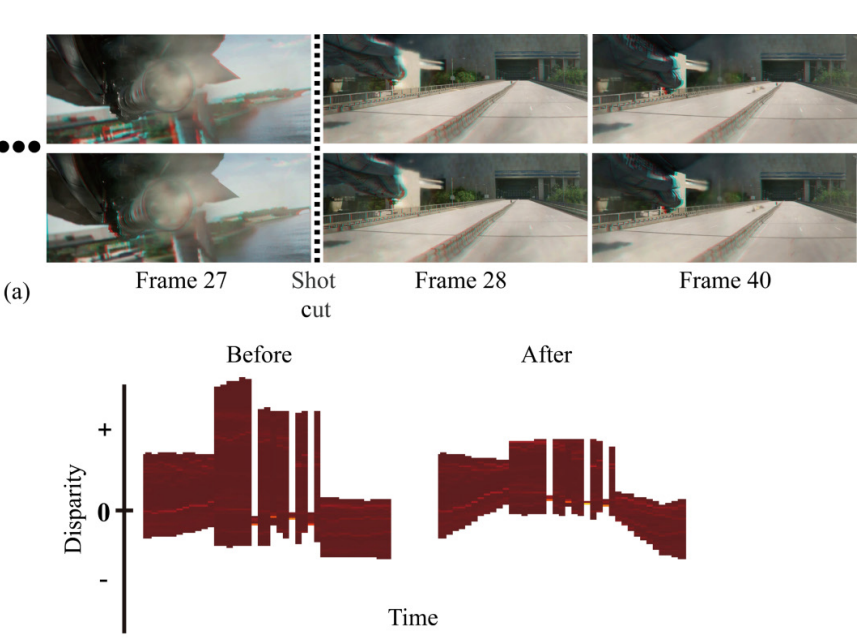

(c)

Fig. 9 Representative anaglyph frames of our results, with a fluent depth storyboard. (a) Sample input and output frames (frame 1 and frame 40 are fixed to their original disparities). (b) Pixel disparity mappings along the time-line (colour encodes output disparity value). (c) Depth storyboard before and after the manipulation, with colour encoding frequency of the occurrence of disparity values. 


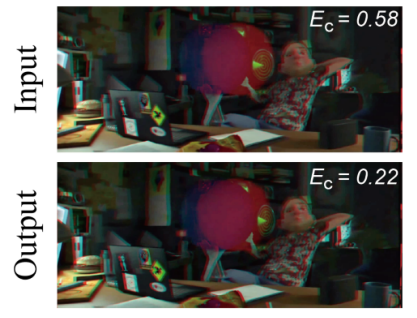

(a)

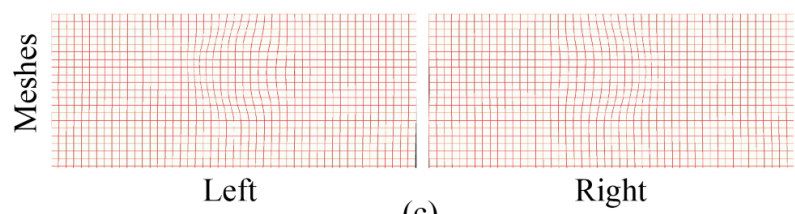

(c)

Fig. 10 Disparity mapping. (a) An input S3D frame and corresponding output frame. The "ball" outside the comfort zone is pushed back towards the screen. (b) Disparity mapping generated by our algorithm. (c) Deformed meshes for left and right views, indicating the warping effect.

after manipulation are 0.58 and 0.22 , respectively.

Figure 11 gives an example of eliminating stereoscopic window violation. In the original input frame, the front of the car appears in front of the screen plane, but is occluded by the picture boundary. This causes the leftmost part of the image to be seen only by the left eye. Such inconsistent content gives an unpleasant viewing experience. Our approach detects such violation automatically and eliminates it by pushing the car behind the screen.

We further tested our framework using videos from a consumer stereoscopic camera (Fuji FinePix REAL 3D W3). Typical frames from one video are shown in Fig. 12. The perceptual depth range is excessive, making it hard to fuse the scene. In the result, the depth of the building in the far distance is reduced, while the disparity values of the flowers are subtly changed.

Perceptual depth enhancement. In Sections 4 and 5 , we presented an extensible framework that optimises disparity mappings driven by comfort. A variation of this framework can be used to drive disparity manipulation to provide

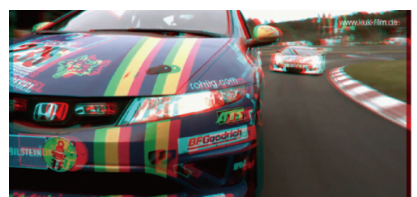

Input frame

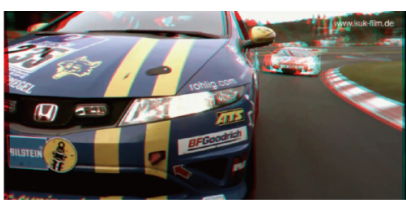

Output frame
Fig. 11 Eliminating stereoscopic window violation. Left: input frames with SWV (c) KUK Filmproduktion $\mathrm{GmbH}$ ). Right: in the manipulation result, the popped out parts are pushed back towards the screen.

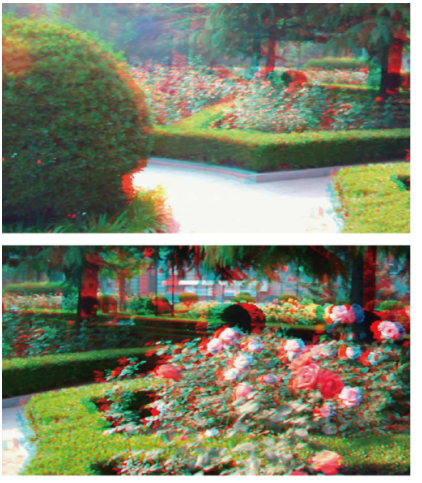

Input frames

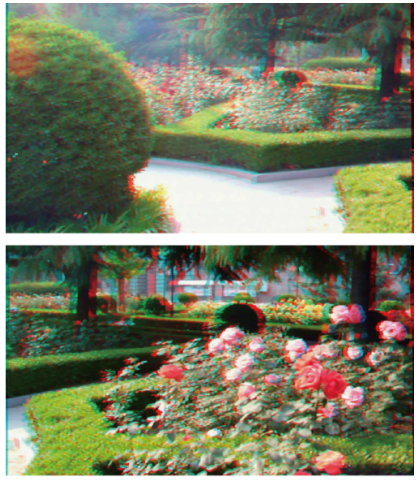

Output frames
Fig. 12 Processing a video taken by a consumer S3D camera. The depth of the scene is reduced to facilitate stereoscopic fusion.

depth enhancement (while not introducing visual discomfort), for a greater feeling of depth. This goal can be accomplished by introducing to the objective function an additional unary term $E_{\mathrm{a}}(\phi, f)$ for each frame with weight $\lambda_{\mathrm{a}}=1$, with the aim of punishing small disparities after applying the mapping $\phi$ to the video:

$$
E_{\mathrm{a}}(\phi, f)=\exp \left(-\frac{1}{2 N} \sum_{d \in\left[d_{\min }, d_{\max }\right]} \Psi_{d}(f)|\phi(d, f)|\right)
$$

where $N$ is the number of pixels in frame $f, d$ is the integer pixel disparity value, and $\Psi_{d}(f)$ is the disparity histogram count for disparity $d$ in frame $f$, as in Eq. (8). This change allows the perceived depths to be amplified, as shown in Fig. 13.

\subsection{User study}

We conducted two user studies with 20 subjects aged from 18 to 32 , to further assess the performance of our proposed comfort-driven disparity adjustment method. The primary aims for the two user studies were to test whether the framework can produce artifact-free results, and its ability to improve visual
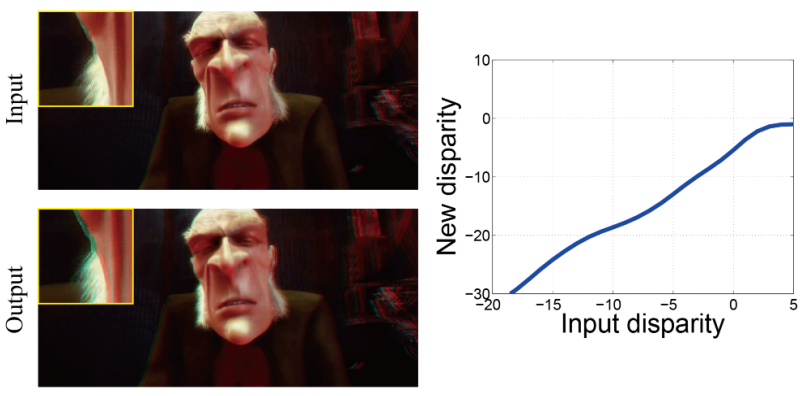

Fig. 13 Enhancing perceptual depth. Left: input and output frames (C) Blender Foundation). After enhancement, the head of the man looks more angular. Right: the generated disparity mapping. 
comfort. Subjects participated by watching S3D videos and filling in questionnaires.

We used a 23-inch interleaved 3D display $\left(1920 \times 1080\right.$ pixels, $400 \mathrm{~cd} / \mathrm{m}^{2}$ brightness $)$, with passive polarized glasses. The viewing distance was set to $55 \mathrm{~cm}$, as assumed in the proposed metric. All subjects had normal or corrected-to-normal vision, and were assessed to ensure they had no difficulty in stereoscopic fusion. Videos were displayed at full screen size.

We prepared ten pairs of S3D videos including animated cartoons and real S3D videos. Both videos in a pair had the same content, one being the original and the other being modified by our system. A random order was used for each pair, and displayed three times in succession. Subjects were allowed to pause and carefully examine the content at any time.

In the first user study, we evaluated whether our output video provides greater visual comfort than the original. After watching each video, each subject was asked to rate the comfort level of their viewing experience, in terms of ease of fusing the scene, causing fewer or more severe headaches, and other feelings of discomfort. Five ratings were used, from 1 to 5: very uncomfortable, uncomfortable, mildly comfortable, comfortable, very comfortable. In all ten pairs of test videos, our results achieved on average a higher comfort score than the original video. The differences in average score in each pair varied from 0.3 to 1.35 . A right-tailed pairedsample hypothesis test was conducted, with the null hypothesis $H_{0}$ : there was no significant difference between the comfort scores of our outputs and the original videos and alternate hypothesis $H_{\mathrm{A}}$ : the comfort scores of our results were significantly higher than those for the original videos at significance level $\alpha=0.05$ with $n=200$ samples. The one-tailed critical value was $t=1.653$, while the test statistic was $t^{*}=9.905$. Since $t^{*}>t$, the null hypothesis was rejected, indicating that the differences were statistically significant: our approach provides an improved stereoscopic video viewing experience.

The second user study aimed to assess artifacts in our results. Before undertaking the user study, the subject was told to note any disturbing perceptual depth artifacts (e.g., depth reversals or unsuitable depth changes) that caused confusion. After watching each video, the subject was asked to rate both videos for unsuitable perceived depths, which were scored as follows: $4=$ many strong artifacts, $2=$ few strong/many weak artifacts, $1=$ few weak artifacts, and $0=$ no artifacts. The results showed that 8 out of 20 subjects did not notice artifacts in any video, 2 subjects only saw artifacts in our results and 2 subjects only saw artifacts in the original videos. The other 8 subjects noticed artifacts in both our results and the original videos. The worst score for both our results and the original videos was 2 (few strong/many weak artifacts). To further test whether statistically the two sets of scores have no difference, a two-tailed paired-sample hypothesis test was conducted, with the null hypothesis $H_{0}$ : there was no significant difference between the artifact scores of our outputs and the original videos and alternate hypothesis $H_{\mathrm{A}}$ : artifact scores of our results and the original videos differ at significance level $\alpha=0.05$ with $n=200$ samples. The two-tailed critical value was $t=1.972$, while the test statistic was $t^{*}=1.236$. This time, the null hypothesis was not rejected, as $\left|t^{*}\right| \leqslant|t|$. We conclude that there is no significant difference in the perceived level of artifacts in the original videos and our results. Indeed, viewers are fairly insensitive to artifacts in these videos. Full statistics of the user studies are provided in the Electronic Supplementary Material (ESM) of this paper.

Limitations. Our approach has limitations. As optimisation is based on a genetic algorithm, it may only find a local optimum. However, tests in which the genetic algorithm was initialized with differing initial populations led to quite similar output mappings. Secondly, existing individual comfort models work well only for viewers with normal stereoscopic fusion ability, and give an average comfort evaluation. Thus using the discomfort metric with default parameters may not give an accurate comfort evaluation for every individual, especially for those with poor stereoscopic fusion ability. Across individuals, there may well be differences in which aspects of an S3D video cause most discomfort. Moreover, our system cannot predict directors' intention: shots intentionally causing discomfort for artistic visual impact would unfortunately be eliminated by our system. 


\section{Conclusions}

We have suggested a general framework for automatic comfort-driven disparity adjustment, together with an S3D discomfort metric. The metric combines several key factors, and could be of general benefit to S3D movie makers by giving an objective visual comfort evaluation in the round. It underpins our automatic disparity adjustment approach, which is based on disparity mapping optimisation. Our results demonstrate the effectiveness and uses of our approach.

Our work is among the first attempts to tackle this challenging problem, and leaves room for improvement. In our framework, the disparity mapping is automatically determined using a heuristic method, and a closed-form solution for this problem is desirable.

\section{Acknowledgements}

This work was supported by the National Hightech R\&D Program of China (Project No. 2013AA013903), the National Natural Science Foundation of China (Project Nos. 61272226 and 61133008), and Research Grant of Beijing Higher Institution Engineering Research Center.

Electronic Supplementary Material Supplementary material is available in the online version of this article at http://dx.doi.org/10.1007/s41095-016-0037-5.

\section{References}

[1] Maimone, A.; Wetzstein, G.; Hirsch, M.; Lanman, D.; Raskar, R.; Fuchs, H. Focus 3D: Compressive accommodation display. ACM Transactions on Graphics Vol. 32, No. 5, Article No. 153, 2013.

[2] Wetzstein, G.; Lanman, D.; Heidrich, W.; Raskar, R. Layered 3D: Tomographic image synthesis for attenuation-based light field and high dynamic range displays. ACM Transactions on Graphics Vol. 30, No. 4, Article No. 95, 2011.

[3] Didyk, P.; Ritschel, T.; Eisemann, E.; Myszkowski, K.; Seidel, H.-P. A perceptual model for disparity. ACM Transactions on Graphics Vol. 30, No. 4, Article No. 96, 2011.

[4] Didyk, P.; Ritschel, T.; Eisemann, E.; Myszkowski, K.; Seidel, H.-P.; Matusik, W. A luminancecontrast-aware disparity model and applications. ACM Transactions on Graphics Vol. 31, No. 6, Article No. $184,2012$.

[5] Hoffman, D. M.; Girshick, A. R.; Akeley, K.; Banks, M. S. Vergence-accommodation conflicts hinder visual performance and cause visual fatigue. Journal of Vision Vol. 8, No. 3, 33, 2008.

[6] Howard, I. P.; Rogers, B. J. Perceiving in Depth, Vol. 2: Stereoscopic Vision. New York: Oxford University Press, 2012.

[7] Palmer, S. E. Vision Science: Photons to Phenomenology. Cambridge, MA, USA: MIT Press, 1999.

[8] Mendiburu, B. 3D Movie Making: Stereoscopic Digital Cinema from Script to Screen. Oxon, UK: Focal Press, 2009.

[9] Kellnhofer, P.; Ritschel, T.; Myszkowski, K.; Seidel, H.-P. Optimizing disparity for motion in depth. Computer Graphics Forum Vol. 32, No. 4, 143-152, 2013.

[10] Lang, M.; Hornung, A.; Wang, O.; Poulakos, S.; Smolic, A.; Gross, M. Nonlinear disparity mapping for stereoscopic 3D. ACM Transactions on Graphics Vol. 29, No. 4, Article No. 75, 2010.

[11] Liu, C.-W.; Huang, T.-H.; Chang, M.-H.; Lee, K.Y.; Liang, C.-K.; Chuang, Y.-Y. 3D cinematography principles and their applications to stereoscopic media processing. In: Proceedings of the 19th ACM International Conference on Multimedia, 253-262, 2011.

[12] Shibata, T.; Kim, J.; Hoffman, D. M.; Banks, M. S. The zone of comfort: Predicting visual discomfort with stereo displays. Journal of Vision Vol. 11, No. 8, 11, 2011.

[13] Cho, S.-H.; Kang, H.-B. Subjective evaluation of visual discomfort caused from stereoscopic 3D video using perceptual importance map. In: Proceedings of IEEE Region 10 Conference, 1-6, 2012.

[14] Du, S.-P.; Masia, B.; Hu, S.-M.; Gutierrez, D. A metric of visual comfort for stereoscopic motion. ACM Transactions on Graphics Vol. 32, No. 6, Article No. 222, 2013.

[15] Jung, Y. J.; Lee, S.-i.; Sohn, H.; Park, H. W.; Ro, Y. M. Visual comfort assessment metric based on salient object motion information in stereoscopic video. Journal of Electronic Imaging Vol. 21, No. 1, 011008, 2012.

[16] Kooi, F. L.; Toet, A. Visual comfort of binocular and 3D displays. Displays Vol. 25, Nos. 2-3, 99-108, 2004.

[17] Mu, T.-J.; Sun, J.-J.; Martin, R. R.; Hu, S.-M. A response time model for abrupt changes in binocular disparity. The Visual Computer Vol. 31, No. 5, 675$687,2015$.

[18] Templin, K.; Didyk, P.; Myszkowski, K.; Hefeeda, M. M.; Seidel, H.-P.; Matusik, W. Modeling and optimizing eye vergence response to stereoscopic cuts. ACM Transactions on Graphics Vol. 33, No. 4, Article No. 145, 2014.

[19] Jin, E. W.; Miller, M. E.; Endrikhovski, S.; Cerosaletti, C. D. Creating a comfortable stereoscopic viewing experience: Effects of viewing distance and field of view on fusional range. In: Proceedings of SPIE 5664, Stereoscopic Displays and Virtual Reality Systems XII, 10, 2005. 
[20] Ukai, K.; Howarth, P. A. Visual fatigue caused by viewing stereoscopic motion images: Background, theories, and observations. Displays Vol. 29, No. 2, 106-116, 2008.

[21] Zilly, F.; Müller, M.; Eisert, P.; Kauff, P. The stereoscopic analyzer - An image-based assistance tool for stereo shooting and 3D production. In: Proceedings of the 17th IEEE International Conference on Image Processing, 4029-4032, 2010.

[22] Masia, B.; Wetzstein, G.; Didyk, P.; Gutierrez, D. A survey on computational displays: Pushing the boundaries of optics, computation, and perception. Computers \& Graphics Vol. 37, No. 8, 1012-1038, 2013.

[23] Lo, W.-Y.; van Baar, J.; Knaus, C.; Zwicker, M.; Gross, M. H. Stereoscopic 3D copy \& paste. ACM Transactions on Graphics Vol. 29, No. 6, Article No. 147, 2010.

[24] Tong, R.-F.; Zhang, Y.; Cheng, K.-L. StereoPasting: Interactive composition in stereoscopic images. IEEE Transactions on Visualization and Computer Graphic Vol. 19, No. 8, 1375-1385, 2013.

[25] Kim, Y.; Lee, Y.; Kang, H.; Lee, S. Stereoscopic 3D line drawing. ACM Transactions on Graphics Vol. 32, No. 4, Article No. 57, 2013.

[26] Niu, Y.; Feng, W.-C.; Liu, F. Enabling warping on stereoscopic images. ACM Transactions on Graphics Vol. 31, No. 6, Article No. 183, 2012.

[27] Kim, C.; Hornung, A.; Heinzle, S.; Matusik, W.; Gross, M. Multi-perspective stereoscopy from light fields. ACM Transactions on Graphics Vol. 30, No. 6, Article No. 190, 2011.

[28] Masia, B.; Wetzstein, G.; Aliaga, C.; Raskar, R.; Gutierrez, D. Display adaptive 3D content remapping. Computers \&f Graphics Vol. 37, No. 8, 983-996, 2013.

[29] Koppal, S. J.; Zitnick, C. L.; Cohen, M.; Kang, S. B.; Ressler, B.; Colburn, A. A viewer-centric editor for 3D movies. IEEE Computer Graphics and Applications Vol. 31, No. 1, 20-35, 2011.

[30] Oskam, T.; Hornung, A.; Bowles, H.; Mitchell, K.; Gross, M. OSCAM-optimized stereoscopic camera control for interactive 3D. ACM Transactions on Graphics Vol. 30, No. 6, Article No. 189, 2011.

[31] Tseng, K.-L.; Huang, W.-J.; Luo, A.-C.; Huang, W.H.; Yeh, Y.-C.; Chen, W.-C. Automatically optimizing stereo camera system based on 3D cinematography principles. In: Proceedings of 3DTV-Conference: The True Vision - Capture, Transmission and Display of 3D Video, 1-4, 2012

[32] Cheng, M.-M.; Mitra, N. J.; Huang, X.; Torr, P. H. S.; Hu, S.-M. Global contrast based salient region detection. IEEE Transactions on Pattern Analysis and Machine Intelligence Vol. 37, No. 3, 569-582, 2015.

[33] Felzenszwalb, P. F.; Huttenlocher, D. P. Efficient graph-based image segmentation. International Journal of Computer Vision Vol. 59, No. 2, 167-181, 2004 .
[34] Achanta, R.; Shaji, A.; Smith, K.; Lucchi, A.; Fua, P.; Süsstrunk, S. SLIC superpixels compared to stateof-the-art superpixel methods. IEEE Transactions on Pattern Analysis and Machine Intelligence Vol. 34, No. 11, 2274-2282, 2012.

[35] Manning, C. D.; Schütze, H. Foundations of Statistical Natural Language Processing. Cambridge, MA, USA: MIT Press, 1999.

[36] Syswerda, G. A study of reproduction in generational and steady state genetic algorithms. Foundation of Genetic Algorithms Vol. 2, 94-101, 1991.

[37] Syswerda, G. Uniform crossover in genetic algorithms. In: Proceedings of the 3rd International Conference on Genetic Algorithms, 2-9, 1989.

[38] Higashi, N.; Iba, H. Particle swarm optimization with Gaussian mutation. In: Proceedings of the IEEE Swarm Intelligence Symposium, 72-79, 2003.

[39] Brox, T.; Malik, J. Large displacement optical flow: Descriptor matching in variational motion estimation. IEEE Transactions on Pattern Analysis and Machine Intelligence Vol. 33, No. 3, 500-513, 2011.

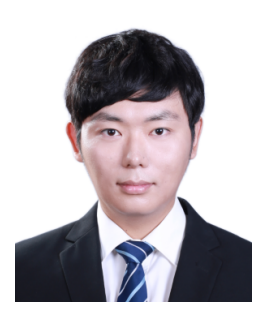

Miao Wang is currently a Ph.D. candidate at Tsinghua University, Beijing, China. He received his B.S. degree from Xidian University in 2011. His research interests include computer graphics, image processing, and computer vision.

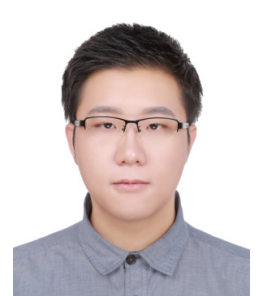

Xi-Jin Zhang is currently a Ph.D. candidate at Tsinghua University, Beijing, China. He received his B.S. degree from Xidian University in 2014. His research interests include image and video processing, computer vision, and machine learning.

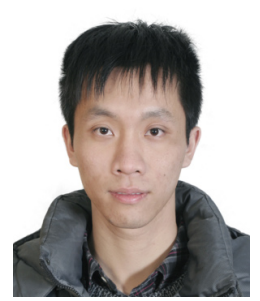

Jun-Bang Liang is currently an undergraduate student at Tsinghua University, Beijing, China. His research interests include computer vision and computer graphics.

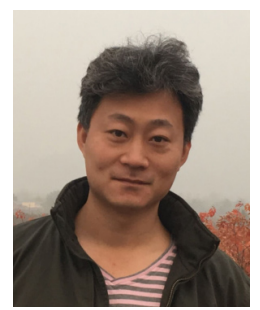

Song-Hai Zhang received his Ph.D. degree in 2007 from Tsinghua University. $\mathrm{He}$ is currently an associate professor in the Department of Computer Science and Technology of Tsinghua University, Beijing, China. His research interests include image and video processing, geometric computing. 


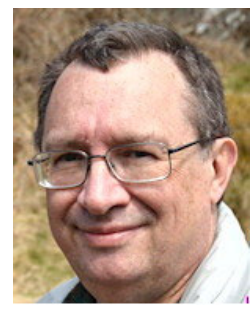

Ralph R. Martin is currently a professor at Cardiff University. $\mathrm{He}$ obtained his Ph.D. degree in 1983 from Cambridge University. He has published about 300 papers and 14 books, covering such topics as solid and surface modeling, intelligent sketch input, geometric reasoning, reverse engineering, and various aspects of computer graphics. $\mathrm{He}$ is a Fellow of the Learned Society of Wales, the Institute of Mathematics and its Applications, and the British Computer Society. $\mathrm{He}$ is on the editorial boards of Computer-Aided Design, Computer Aided Geometric Design, Geometric Models, and Computers and Graphics.
Open Access The articles published in this journal are distributed under the terms of the Creative Commons Attribution 4.0 International License (http:// creativecommons.org/licenses/by/4.0/), which permits unrestricted use, distribution, and reproduction in any medium, provided you give appropriate credit to the original author(s) and the source, provide a link to the Creative Commons license, and indicate if changes were made.

Other papers from this open access journal are available free of charge from http://www.springer.com/journal/41095. To submit a manuscript, please go to https://www. editorialmanager.com/cvmj. 